CONGENITAL HEART DISEASE

\title{
Persistent pulmonary hypertension late after neonatal aortic valvotomy: a consequence of an expanded surgical cohort
}

\author{
M Burch, L Kaufman, N Archer, I Sullivan
}

Heart 2004;90:918-920. doi: 10.1136/hrt.2003.024760

See end of article for authors' affiliations

.....................

Correspondence to: Dr Michael Burch, Great Ormond Street Hospital for Children, Great Ormond Street, London WCIN 3JH, UK; burchm@gosh. nhs.uk

Accepted

16 December 2003

\begin{abstract}
Background: Survival of neonates with critical aortic stenosis has improved over the past decade. Models based on morphological characteristics have been designed to help determine whether early survival is more likely after biventricular repair (surgical or balloon aortic valvotomy) or after a single ventricle staged palliative surgical strategy. However, late follow up data are lacking.

Objective: To report follow up data on survivors of neonatal aortic valvotomy who had persistent pulmonary hypertension caused by restriction to left ventricular filling.

Results: Of four medium term survivors of neonatal valvotomy for critical aortic stenosis who had persistent pulmonary hypertension, one died aged 4 years and the other three have severe limitation of effort tolerance.

Conclusions: This previously unreported late complication of "successful" biventricular repair for neonatal critical aortic stenosis is an important consideration in determining the initial management.
\end{abstract}

$\mathrm{T}$ he outcome for neonates born with critical aortic valve stenosis has improved over recent years, but there continues to be a high attrition rate. Hospital survival was $48 \%$ in 73 neonates who underwent intervention at a mean age of eight days over two decades (1970 to 1993) at two institutions. ${ }^{1}$ Subsequent experience has been more favourable, with $77 \%$ survival at age 3 months and $70 \%$ at 5 years after biventricular repair at 24 institutions in the era 1994 to 2000. ${ }^{2}$ Outcome was similar for either balloon or surgical valvotomy. ${ }^{3}$ Consequently, there is an expanded pool of late survivors. The long term prognosis of these children is unknown. We report here four children born with critical aortic stenosis who had persisting pulmonary hypertension secondary to restriction of left ventricular filling, a previously unreported long term complication of apparently successfully treated neonatal critical aortic stenosis.

\section{CASE REPORTS}

\section{Case 1}

Heart disease was diagnosed at 33 weeks' gestation by prenatal ultrasound. There was severe aortic stenosis, mild aortic incompetence, severe mitral regurgitation, and poor function of the left ventricle with bright left ventricular myocardial echoes. Labour was induced at 38 weeks' gestation. The female infant underwent aortic valvotomy on cardiopulmonary bypass on day 3. Echocardiography at five weeks showed improved left ventricular function with only mild mitral regurgitation and mild aortic incompetence. The maximum systolic velocity across the aortic valve was $1.9 \mathrm{~m} / \mathrm{s}$. There was persistence of high peak velocity tricuspid regurgitation. Cardiac catheterisation at age 6 months confirmed pulmonary hypertension with a pulmonary artery wedge pressure of $20 \mathrm{~mm} \mathrm{Hg}$ and a left ventricular end diastolic pressure of $19 \mathrm{~mm} \mathrm{Hg}$. A second catheterisation at 17 months of age showed no left ventricular outflow tract obstruction, mild mitral and aortic regurgitation, no pulmonary vein stenosis, and suprasystemic pulmonary artery pressure (table 1). Sequential echocardiography revealed increasing right ventricular pressures with right ventricular dilatation. Severe failure to thrive continued. Acute deterioration and death occurred when she was 4 years old.

\section{Case 2}

A female infant born at term presented at 2 weeks of age with no palpable arterial pulses and severely compromised systemic blood flow. Echocardiography showed severe aortic stenosis with trivial aortic regurgitation. Aortic valvotomy was undertaken using cardiopulmonary bypass. Echocardiography two weeks later showed improved left ventricular function, peak velocity in the ascending aorta of $1.6 \mathrm{~m} / \mathrm{s}$, mild aortic regurgitation, and no mitral regurgitation. The right ventricle was dilated but no tricuspid regurgitation was detected. Subsequent progress was characterised by poor growth, and Turner's syndrome (45XO karyotype) was diagnosed. Serial echocardiograms showed left ventricular hypertrophy, no residual aortic stenosis (ascending aorta peak flow velocity $1.9 \mathrm{~m} / \mathrm{s}$ ), moderate aortic regurgitation, and good left ventricular systolic function. The right ventricle remained dilated. Cardiac catheterisation and angiography at age 9 years confirmed pulmonary hypertension (table 1) and moderate aortic regurgitation. Aortic valve replacement was undertaken with a $21 \mathrm{~mm}$ Aortech valve. Now aged 12 years she continues to have limited effort tolerance. A recent echocardiogram showed severe enlargement of the right atrium and right ventricle. Estimated right ventricular systolic pressure was $67 \mathrm{~mm} \mathrm{Hg}$. Mild paraprosthetic aortic regurgitation was seen, with a low end diastolic peak velocity $(1.6 \mathrm{~m} / \mathrm{s})$, indicating severely increased left ventricular end diastolic pressure.

\section{Case 3}

This male infant was the product of a twin pregnancy, born at 38 weeks' gestation, birth weight $2.2 \mathrm{~kg}$. He presented at 2 weeks of age with severe cardiac failure. Echocardiography showed concentric left ventricular hypertrophy, poor left ventricular function, and severe aortic stenosis with a Doppler estimated systolic pressure drop of $80 \mathrm{~mm} \mathrm{Hg}$. Open aortic valvotomy using cardiopulmonary bypass was done at 16 days of age. Initially progress was good, but by 2 years he was breathless on exertion, with poor weight gain. On echocardiography the left atrium was enlarged, there was residual aortic stenosis with a Doppler gradient of $50 \mathrm{~mm} \mathrm{Hg}$, and moderate aortic regurgitation. Autograft replacement of the aortic root (Ross operation) and mitral valve inspection 
Table 1 Cardiac catheterisation details

\begin{tabular}{|c|c|c|c|c|c|c|c|}
\hline \multirow[b]{2}{*}{ Patient number } & \multirow[b]{2}{*}{ Year of birth } & \multirow{2}{*}{$\begin{array}{l}\text { Age at first surgery } \\
\text { (days) }\end{array}$} & \multicolumn{4}{|c|}{ Catheter data } & \multirow[b]{2}{*}{ Outcome } \\
\hline & & & PA & PCW & LVEDP & Age (years) & \\
\hline 1 & 1997 & 3 & 63 & 34 & 40 & 1 & Died at 4 years \\
\hline 2 & 1991 & 15 & 60 & 30 & 30 & 9 & NYHA III \\
\hline 3 & 1993 & 16 & 45 & 23 & 26 & 5 & NYHA III \\
\hline 4 & 1995 & 2 & 48 & 22 & 31 & 6 & NYHA III \\
\hline
\end{tabular}

LVEDP, left ventricular end diastolic pressure ( $\mathrm{mm} \mathrm{Hg}$ ); NYHA, New York Heart Association; PA, mean pulmonary artery pressure (mm Hg); PCW, mean pulmonary capillary wedge pressure $(\mathrm{mm} \mathrm{Hg})$.

was undertaken. At follow up there was persisting pulmonary hypertension and development of mild left ventricular inflow obstruction. At age 4 years, syncope preceded by abdominal pain occurred on three occasions. Cardiac catheterisation showed slightly subsystemic pulmonary artery pressure and an end diastolic gradient of $6 \mathrm{~mm} \mathrm{Hg}$ between indirect left atrial and left ventricular pressures. Reoperation was carried out. Both mitral valve papillary muscles were hypertrophied, with short chordae. The papillary muscles were mobilised by dividing adherent fibrotic trabeculations. At the end of the procedure, left atrial mean pressure was $11 \mathrm{~mm} \mathrm{Hg}$ and left ventricular end diastolic pressure was $21 \mathrm{~mm} \mathrm{Hg}$. Left ventricular biopsy showed notable fibroelastosis with thickening of the endocardium (fig 1). There was symptomatic improvement, with no further syncope, but poor growth and limited effort tolerance has persisted. Cardiac catheterisation was done most recently at the age of five years (table 1).

\section{Case 4}

Aortic stenosis was diagnosed at 20 weeks' gestation. The left ventricular cavity was of normal size, but there were bright myocardial echoes, most notably around the left ventricular free wall and the septal surface of the left ventricle (fig 2), and impaired systolic function. There was retrograde flow in the aortic arch, indicating right ventricle dependent flow through the arterial duct. Against expectation, at 31 weeks' gestation there was improvement in left ventricular function with restoration of anterograde flow in the aortic arch. Normal delivery occurred at 39 weeks' gestation, with a birth weight of $2.4 \mathrm{~kg}$. Surgical valvotomy was undertaken on the second day of life, using cardiopulmonary bypass. He made an uneventful recovery. However, at follow up there was poor growth, limited effort capacity, episodes suggesting

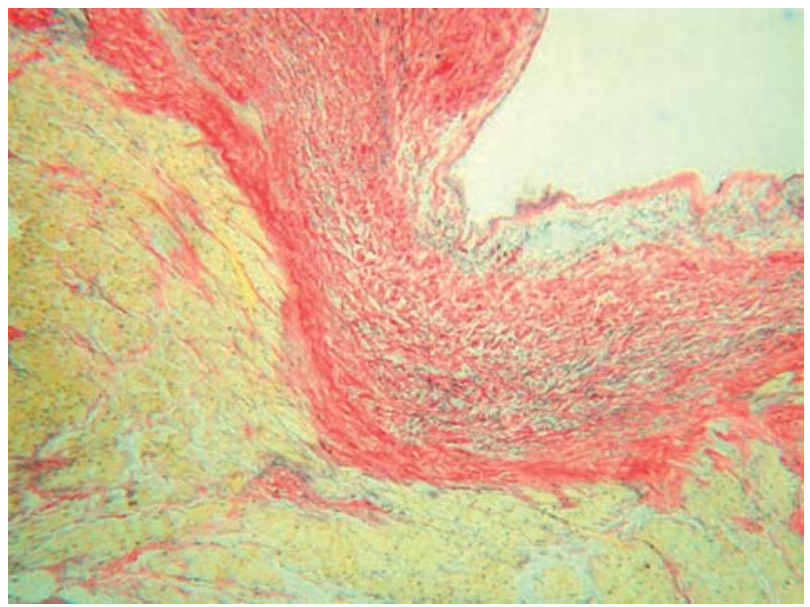

Figure 1 Light microscopy from operative myocardial biopsy in case 3 showing notable fibroelastic thickening of the left ventricular endocardium. (By courtesy of Dr A Ramsay.) presyncope, and features of pulmonary hypertension. Cardiac catheterisation was done at 6 years of age (table 1). Echocardiography showed that the right ventricle was enlarged and the tricuspid regurgitation velocity confirmed increased right ventricular systolic pressure. The left ventricular cavity was of normal size with good systolic function, but had persistence of bright myocardial echoes. Peak systolic flow velocity in the ascending aorta was $2.7 \mathrm{~m} / \mathrm{s}$ and there was moderate aortic regurgitation. It was felt that further aortic valve surgery was not indicated. Treatment with $\beta$ blockade and angiotensin converting enzyme inhibition was begun, but there had been no improvement in the haemodynamic measurements when cardiac catheterisation was repeated seven months later.

\section{DISCUSSION}

The most comprehensive data available on early survival of neonates born with critical aortic valve stenosis are those reported from the Congenital Heart Surgeons Society study: an initial "biventricular repair", essentially balloon or open aortic valvotomy, was done in 116 patients, and an initial Norwood operation in $179 .^{2}$ Mathematical models for survival were calculated for each pathway and then used to predict the optimal pathway for each patient, based on functional and morphological features. Not surprisingly, the neonates entered on the biventricular repair pathway had greater aortic valve, aortic root, and mitral valve indexed diameters than the Norwood cohort. Surprising, perhaps, was a predicted five year survival benefit favouring the Norwood pathway in 50\% of those who had biventricular repair. Conversely, biventricular repair was predicted as more favourable in $20 \%$ of those neonates who underwent the Norwood procedure. The main risk factors for death after aortic valvotomy were young age, a small aortic valve, a short left ventricle, and a high grade of

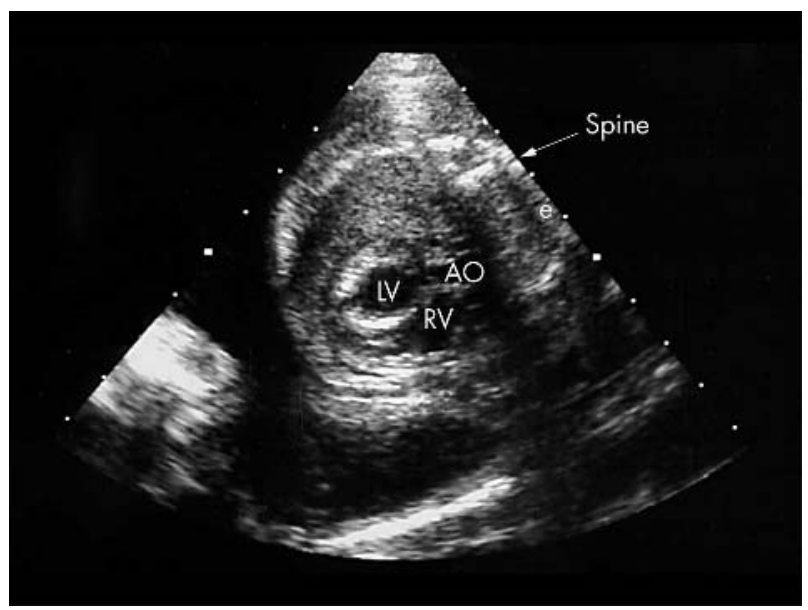

Figure 2 Fetal echocardiography in case 4: transverse thoracic view showing bright endocardial echoes. The spine is labelled for orientation. $\mathrm{AO}$, aorta; LV, left ventricle; RV, right ventricle. 
"endocardial fibroelastosis". The last of these was graded subjectively by echocardiography, but this grading correlated only weakly with necropsy grading of endocardial fibroelastosis in the hearts that were available for comparison. This suggests that it should not be assumed that bright endocardial echoes necessarily reflect the same process, even if this appearance provides useful prognostic information.

Late follow up data after neonatal interventions for critical aortic stenosis are lacking. Our previous experience was that hospital survivors of aortic valvotomy had reasonable medium term survival (93\% at 10 years, $84 \%$ at 15 years) with a good functional result, though often with the need for further left ventricular outflow tract surgery. ${ }^{1}$ We had not recognised late pulmonary hypertension in survivors of neonatal intervention for critical aortic stenosis at the time of that report. Similar medium term (more than five years) follow up data were reported for neonates operated in the same era. ${ }^{45}$

Left ventricular outflow tract obstruction in fetal life is known to cause endocardial fibroelastosis, ${ }^{67}$ with increased production of collagen and elastic fibres. There may also be other myocardial changes such as fibrosis, necrosis, or calcification, perhaps explaining some of the discrepancy between bright left ventricular endocardial echoes and the histological diagnosis of endocardial fibroelastosis. Almost certainly, an increasing number of neonates with these myocardial changes in the context of critical aortic valve stenosis have been surviving neonatal interventions over the past decade.

The likely explanation for the severe pulmonary hypertension persisting up to 12 years after neonatal aortic valve surgery in the patients in this report is restriction to left ventricular filling. Bright left ventricular endocardial echoes were seen prenatally in the two patients who underwent prenatal echocardiography. Endocardial fibroelastosis was confirmed by left ventricular myocardial biopsy in a third patient. Pulmonary hypertension is a well known consequence of restrictive cardiomyopathy ${ }^{8}$ and isolated endocardial fibroelastosis." Interestingly, isolated endocardial fibroelastosis is now rarely seen, possibly because of a link between this condition and the mumps virus, which is now uncommon since the introduction of the MMR vaccine..$^{10}$

The recent data referred to above have provided information about the interventional strategy (aortic valvotomy versus Norwood procedure) that is most likely to result in survival up to five years after neonatal intervention for critical aortic valve stenosis. ${ }^{23}$ However, it was acknowledged that these data could not yet examine long term complications. $^{3}$ Late problems occur with a functionally single ventricle surgical strategy, even in patients with a good medium term outcome. In those who undergo biventricular repair, the likely need for further intervention because of recurrent left ventricular outflow obstruction or aortic regurgitation is well known. Restriction to left ventricular filling secondary to altered heart muscle physiology may be an additional important determinant of the long term outcome. Further information about this previously unreported late complication of "biventricular repair" may help to inform the decision about whether to use neonatal aortic valvotomy or the Norwood operation, and may also lead to consideration of additional or alternative surgical approaches such as left ventricular endocardial resection and autograft replacement of the aortic root, or transplantation, in selected patients.

\section{Authors' affiliations}

M Burch, I Sullivan, Great Ormond Street Hospital for Children, London, UK

L Kaufman, N Archer, John Radcliffe Hospital, Oxford, UK

\section{REFERENCES}

1 Gaynor JW, Bull C, Sullivan ID, et al. Late outcome of survivors of intervention for neonatal aortic stenosis. Ann Thorac Surg 1995;60:122-5.

2 Lofland GK, McCrindle BW, Williams WG, et al. Critical aortic stenosis in the neonate: a multi-institutional study of management, outcomes and risk factors. Congenital Heart Surgeons Society. J Thorac Cardiovasc Surg 2001;121:10-27.

3 McCrindle BW, Blackstone EH, Williams WG, et al. Are outcomes of surgical versus transcatheter balloon valvotomy equivalent in neonatal critical aortic stenosis? Circulation 2001;104(suppl 1):1152-8.

4 Ettedgui JA, Tallman-Eddy T, Neches WH, et al. Long term results of survivors of surgical valvotomy for severe aortic stenosis in early infancy. J Thorac Cardiovasc Surg 1992;104:1714-20.

5 Pelech AN, Dyck JD, Trusler GA, et al. Critical aortic stenosis. Survival and management. J Thorac Cardiovasc Surg 1987;94:510-17.

6 Mielke G, Mayer R, Hassberg D, et al. Sequential development of fetal aortic valve stenosis and endocardial fibroelastosis during the second trimester of pregnancy. Am Heart J 1997; 133:607-10.

7 Sharland GK, Chita SK, Fagg NL, et al. Left ventricular dysfunction in the fetus: relation to aortic valve anomalies and endocardial fibroelastosis. Br Heart J $1991 ; 66: 419-24$

8 Chen SC, Balfour IC, Jureidini S. Clinical spectrum of restrictive cardiomyopathy in children. J Heart Lung Transplant 2001;20:90-2.

9 Mcloughlin TG, Schiebler GL, Krovetz L. Hemodynamic findings in children with endocardial fibroelastosis. Analysis of 22 cases Am Heart $J$ 1968;75:162-72.

$10 \mathrm{Ni}$ J, Bowles NE, Kim YH, et al. Viral infection of the myocardium in endocardial fibroelastosis. Molecular evidence for the role of mumps virus as an etiologic agent. Circulation 1997;95:133-9. 